\section{Perioperative nutritional intervention: a way to improve long-term outcomes}

\author{
Alessio Molfino and Alessandro Laviano
}

We read with interest the recent Review article by Anandavadivelan and Lagergren (Cachexia in patients with oesophageal cancer. Nat. Rev. Clin. Oncol. http://dx.doi.org/10.1038/ nrclinonc.2015.200 (2015)) $)^{1}$, which addressed the important issue of cachexia in patients with oesophageal cancer. Therein, the authors reviewed different pathophysiological and clinical aspects of cachexia in patients with cancer, and concluded that a need exists for additional studies evaluating the efficacy of therapeutic interventions aimed at improving the quality of life of patients - which often declines after major surger $y^{2}-$ by reducing muscle loss and improving muscle function $^{1}$. We agree that achieving these aims is an important goal of nutritional intervention; however, we believe that the real potential of nutritional therapy in patients with cancer stretches beyond mere improvements of body composition and physical performance.

Increasing evidence indicates that interventions made in the perioperative period can be pivotal in determining the long-term outcomes of patients with cancer; modulation of nutritional and metabolic status in the perioperative period is widely acknowledged to reduce the incidence of adverse events and improve surgery-related outcomes ${ }^{3}$. Moreover, alterations of immune function during the perioperative period seem to be a key determinant of outcome, and might even promote, or at least permit, metastatic progression $^{3}$. van Bokhorst-De Van Der Schueren et al. ${ }^{4}$ have published data demonstrating that L-arginine-enriched preoperative enteral nutrition did not improve nutritional status or reduce immunosuppression in severely malnourished patients with head and neck cancer, compared with nutritional support without L-arginine supplementation; nevertheless, the group of patients who received L-arginineenriched nutrition tended to live longer, although the difference did not reach the statistical significance $(P=0.15)$. Further evidence confirmed positive effects of additional
L-arginine supplementation in nutritional support on the long-term disease-specific $(P=0.022)$ and overall survival $(P=0.019)$ of malnourished patients with head and neck cancer ${ }^{5}$; moreover, significantly better locoregional recurrence-free survival was observed among the L-arginine-supplemented patients $(P=0.027)^{5}$. Additional findings have indicated that $\mathrm{L}$-arginine supplementation in the perioperative can, in fact, improve restoration of the immune system and ameliorate immune function ${ }^{6,7}$, and thus could potentially reduce the growth of cancer cells and thereby prolong patient survival - although additional studies are required to confirm this possibility.

The metabolic and nutritional status of patients with oesophageal cancer is often altered because of mechanical obstruction (dysphagia) associated with this disease, and can also be worsened by several adverse effects of chemoradiotherapy - including mucositis ${ }^{8}$. Moreover, protein-energy malnutrition has a negative effect on immune function $^{9,10}$. In 2015, a double-blind, randomized clinical trial in patients with head and neck or oesophageal cancer showed that 'immunonutrition', including L-arginine, was able to modulate the phenotypes and functions of immune cells, and thus enhance immune responses ${ }^{11}$. By modulating the gene-expression profiles of immune cells, immunonutrition might facilitate adaption of the cells to oxidative stress and inflammation induced by chemoradiotherapy, which is one of the most-common postoperative therapeutic options for patients with various tumour types, particularly oesophageal cancer ${ }^{11}$. In turn, such adaptation of immune responses during the crucial perioperative period might negate immunosuppression, and thus limit tumour growth and metastatic dissemination.

Evidence indicates that therapeutic interventions aimed at improving nutritional status, and thereby enhancing immune function, are key determinants of the long-term outcomes of patients with cancer. In the setting of oesophageal cancer, counteracting skeletal muscle loss and improving physical performance are clinically relevant goals of nutritional support; perioperative intervention with tailored nutritional programmes seems to be essential to improve therapeutic responses and survival. Additional data are required to better define the effects of nutrition at the molecular and cellular levels, as well as to clarify the link between these effects and long-term outcomes.

Alessio Molfino and Alessandro Laviano are at the Department of Clinical Medicine, Sapienza University of Rome, Viale dell'Università 37, 00185 Rome, Italy.

Correspondence to A.M. alessio.molfino@uniroma1.it doi: 10.1038/nrclinonc.2016.20 Published online 16 Feb 2016

1. Anandavadivelan, P. \& Lagergren, P. Cachexia in patients with oesophageal cancer. Nat. Rev. Clin. Oncol. http://dx.doi.org/10.1038/nrclinonc.2015.200 (2015).

2. Lagergren, J. \& Lagergren, P. Recent developments in esophageal adenocarcinoma. CA Cancer J. Clin. 63, 232-248 (2013).

3. Horowitz, M., Neeman, E., Sharon, E. \& Ben-Eliyahu, S. Exploiting the critical perioperative period to improve Iong-term cancer outcomes. Nat. Rev. Clin. Oncol. 12 213-226 (2015)

4. van Bokhorst-De Van Der Schueren, M. A. et al. Effect of perioperative nutrition, with and without arginine supplementation, on nutritional status, immune function, postoperative morbidity, and survival in severely malnourished head and neck cancer patients. Am. J. Clin. Nutr. 73, 323-332 (2001).

5. Buijs, N. et al. Perioperative arginine-supplemented nutrition in malnourished patients with head and neck cancer improves long-term survival. Am. J. Clin. Nutr. 92, 1151-1156 (2010).

6. Angele, M. K. et al. L-Arginine: a unique amino acid for restoring the depressed macrophage functions after trauma-hemorrhage. J. Trauma 46, 34-41 (1999).

7. Angele, M. K. et al. L-Arginine restores splenocyte functions after trauma and hemorrhage potentially by improving splenic blood flow. Am. J. Physiol. 276 C145-C151 (1999).

8. Kubrak, C. et al. Clinical determinants of weight loss in patients receiving radiation and chemoirradiation for head and neck cancer: a prospective longitudinal view. Head Neck 35, 695-703 (2013).

9. Langer, C. J., Hoffman, J. P. \& Ottery, F. D. Clinical significance of weight loss in cancer patients: rationale for the use of anabolic agents in the treatment of cancer-related cachexia. Nutrition 17, S1-S20 (2001).

10. Laviano, A. et al. Beyond anorexia-cachexia. Nutrition and modulation of cancer patients' metabolism: supplementary, complementary or alternative antineoplastic therapy? Eur. J. Pharmacol. 668, S87-S90 (2011).

11. Talvas, J. et al. Immunonutrition stimulates immune functions and antioxidant defense capacities of leukocytes in radiochemotherapy-treated head and neck and esophageal cancer patients: a double-blind randomized clinical trial. Clin. Nutr. 34, 810-817 (2015).

\section{Competing interests statement}

A.L. declares that he has received honoraria from Fresenius Kabi, Nutricia, and Smartfish for independent lectures he has given at industry-sponsored events. A.M. declares no competing interests. 